\title{
Niacin Cures Systemic NAD(+) Deficiency and Improves \\ Muscle Performance in Adult-Onset Mitochondrial Myopathy
}

\section{Pirinen, Eija}

2020-06-02

Pirinen , E, Auranen , M , Khan , N A, Brilhante , V, Urho, N, Pessia , A, Hakkarainen , A , Kuula , J , Heinonen, U , Schmidt , M S , Haimilahti , K, Piirilä, P, Lundbom , N , Taskinen , M-R , Brenner , C , Velagapudi , V , Pietiläinen , K H \& Suomalainen , A 2020 , ' Niacin Cures Systemic NAD(+) Deficiency and Improves Muscle Performance in Adult-Onset Mitochondrial Myopathy ' , Cell Metabolism , vol. 31 , no. 6 , pp. 1078-1090 . https://doi.org/10.1016/j.cmet.2020.04.0

http://hdl.handle.net/10138/330502

https://doi.org/10.1016/j.cmet.2020.04.008

cc_by_nc_nd

acceptedVersion

Downloaded from Helda, University of Helsinki institutional repository.

This is an electronic reprint of the original article.

This reprint may differ from the original in pagination and typographic detail.

Please cite the original version. 
Niacin cures systemic NAD + deficiency and improves muscle performance in adultonset mitochondrial myopathy

Eija Pirinen $1^{*}$, Mari Auranen2,3\#, Nahid A. Khan2\#, Virginia Brilhante2, Niina Urho3, Alberto Pessia4, Antti Hakkarainen5,6, Juho Kuula5, Ulla Heinonen3, Mark S. Schmidt7, Kimmo Haimilahti1, Päivi Piiriä8, Nina Lundbom5, Marja-Riitta Taskinen1, Charles Brenner7, Vidya Velagapudi4€, Kirsi H. Pietiläineng,10 and Anu Suomalainen2,11*

1Research Program for Clinical and Molecular Metabolism, Faculty of Medicine, University of Helsinki, FIN-00290 Helsinki, Finland

2Research Program of Stem Cells and Metabolism, Faculty of Medicine, University of Helsinki, FIN-00290 Helsinki, Finland

3Department of Neurosciences, Helsinki University Hospital, Helsinki, Finland

4Metabolomics Unit, Institute for Molecular Medicine Finland (FIMM), FIN-00290 Helsinki, Finland

5Department of Radiology, Medical Imaging Center, University of Helsinki and Helsinki University Hospital, Helsinki, Finland

${ }_{6}$ Department of Neuroscience and Biomedical Engineering, Aalto University School of Science, FIN-12200 Espoo, Finland

7Department of Biochemistry, Carver College of Medicine, University of lowa, lowa City, lowa 52242, USA

${ }_{8}$ Unit of Clinical Physiology, Helsinki University Hospital and University of Helsinki, Helsinki, Finland

9Obesity Research Unit, Research Program for Clinical and Molecular Metabolism, Faculty of Medicine, University of Helsinki, FIN-00290 Helsinki, Finland

10Obesity Centre, Abdominal Centre, Endocrinology, Helsinki University Hospital and University of Helsinki, Helsinki, Finland

11Lead Contact

\#equal contribution

€current affiliation: Biopharmaceuticals R\&D, Discovery Sciences, Astra Zeneca, Gothenburg, Sweden

${ }^{*}$ Correspondence:

anu.wartiovaara@helsinki.fi, eija.pirinen@helsinki.fi 


\section{Summary}

$\mathrm{NAD}_{+}$is a redox-active metabolite, the depletion of which has been proposed to promote aging and degenerative diseases in rodents. However, whether NAD+ depletion occurs in patients with degenerative disorders and whether NAD+ repletion improves their symptoms, has remained open. Here, we report systemic NAD+ deficiency in adult-onset mitochondrial myopathy patients. We administered an increasing dose of NAD+-booster niacin, a vitaminB3 form (to 750-1000 mg/day; clinicaltrials.gov NCT03973203) for patients and their matched controls for 10 or 4 months, respectively. Blood NAD+ increased in all subjects, up to 8-fold, and muscle NAD+ of patients reached the level of their controls. Some patients showed anemia tendency, while muscle strength and mitochondrial biogenesis increased in all subjects. In patients, muscle metabolome shifted towards controls and liver fat decreased even $50 \%$. Our evidence indicates that blood analysis is useful in identifying NAD+-deficiency and points niacin to be an efficient NAD+-booster for treating mitochondrial myopathy.

\section{Keywords}

Niacin, $\mathrm{NAD}_{+}$, vitamin $\mathrm{B} 3, \mathrm{NAD}_{+}$repletion, mitochondrial myopathy, mtDNA deletions, mitochondria, treatment. 


\section{Introduction}

$\mathrm{NAD}_{+}$metabolite and its derivatives are fundamental orchestrators of daily homeostasis in our tissues. The relative amounts of NAD forms (NAD+, NADH, NADP, NADPH) and their cofactor functions to drive metabolism to either catabolic or anabolic direction, deciding whether nutrients are broken down to synthesize ATP, the cellular energy currency (Nunnari and Suomalainen, 2012), or used as building-blocks for growth and repair. The different NAD forms, their ratio and derived metabolites regulate lipid, nucleotide, glutathione synthesis and membrane homeostasis (Belenky et al., 2007a; Canto et al., 2015). A decreased $\mathrm{NAD}_{+} / \mathrm{NADH}$ ratio is a signal for a low nutrient state activating cellular fasting responses (Canto et al., 2015). These responses have been associated with health benefits and longevity. Not surprisingly, NAD+ metabolism has been a key interest of aging biology and therapeutic strategies for degenerative diseases. However, evidence of NAD+ deficiency and effects of NAD + repletion in degenerative conditions relies on data in rodents and cell culture models, but human evidence is lacking.

Mitochondrial activity is the key regulator of $\mathrm{NAD+} / \mathrm{NADH}$ ratio, raising the question whether mitochondrial dysfunction and disease can affect intracellular NAD+ concentration. Mitochondrial disorders are exceptionally variable in manifestations, ranging from severe childhood brain disorders to adult-onset mitochondrial myopathies (Suomalainen and Battersby, 2017), the latter being the most common manifestation of the disease group in adults. Mitochondrial myopathy manifests typically as progressive weakness of the eye muscles [progressive external ophthalmoplegia (PEO)], generalized muscle weakness and fatigability (Ylikallio and Suomalainen, 2012). PEO is often caused by single heteroplasmic mitochondrial DNA (mtDNA) deletions or multiple mtDNA deletions, the former being sporadic and latter caused by mutations in nuclear-encoded proteins of mtDNA maintenance (Holt et al., 1988; Kaukonen et al., 2000; Spelbrink et al., 2001; Suomalainen et al., 1992; 
Van Goethem et al., 2001; Zeviani et al., 1989). Despite recent advances in diagnostics and understanding of molecular mechanisms of PEO, curative interventions do not exist.

Mouse models for some progressive muscle diseases, including mitochondrial myopathy, manifest NAD+ depletion (Khan et al., 2014; Ryu et al., 2016; Zhang et al., 2016). Such a disease-modified, nutrient-independent $\mathrm{NAD}_{+}$deficiency may disturb nutrient signaling in tissues and contribute to disease progression (Nunnari and Suomalainen, 2012). Therefore, the finding that increasing NAD+ levels remarkably improved disease hallmarks, mitochondrial mass and biogenesis in mitochondrial myopathy mice was highly interesting considering therapeutic approaches (Cerutti et al., 2014; Khan et al., 2014). Intracellular $N A D_{+}$concentrations can be increased by various approaches, such as decreasing NAD+ consumption (inhibition of poly(ADP-ribose) polymerases and cluster-of-differentiation-38) (Canto et al., 2015; Pirinen et al., 2014), NAD+ precursor supplementation (Bieganowski and Brenner, 2004; Canto et al., 2015), or by inhibition of aminocarboxymuconate semialdehyde decarboxylase (Katsyuba et al., 2018), which results in increased de novo synthesis of NAD+ from tryptophan. Vitamin $\mathrm{B} 3$ is an $\mathrm{NAD}_{+}$precursor, and exists in several forms: nicotinic acid (niacin), nicotinamide (NAM), and nicotinamide riboside (NR) (Belenky et al., 2007b; Canto et al., 2015). In two mouse models for mitochondrial myopathy, NR remarkably improved mitochondrial function and mass, and delayed disease symptoms (Cerutti et al., 2014; Khan et al., 2014). However, studies of the effects of NAD+ boosters on human diseases are in their infancy.

Here, we determined NAD+ metabolome and the effects of niacin on NAD+ levels and disease signs in mitochondrial myopathy patients and controls. Our main question was whether NAD+ levels are depleted in mitochondrial dysfunction, as mitochondria are regulating NAD+ concentrations, and if so, whether NAD+ deficiency can be restored in the tissues of the patients. Niacin was employed, because it has been used in large doses to 
treat hypercholesterolemia patients for the past 50 years, and has a proven safety record in humans (Guyton and Bays, 2007). Despite the long use of niacin for dyslipidemia, the mechanism of action of niacin is not clear and it was only recently proposed to involve elevation of NAD+ (Belenky et al., 2007a). Here we show that mitochondrial myopathy patients have depressed systemic NAD+ metabolism that can be functionally addressed with a high dose of niacin.

\section{Results}

\section{Niacin is well tolerated by study subjects}

We recruited phenotypically similar mitochondrial myopathy patients for our study, as our previous expertise indicated that similar presenting phenotypes predict uniform physiological responses to interventions, despite varying genetic backgrounds (Ahola et al., 2016). Five patients with variable disease duration were carefully confirmed to manifest a pure mitochondrial myopathy, with PEO, ptosis, muscle weakness and exercise intolerance. They all carried either heteroplasmic single or multiple mtDNA deletions in their muscle. Table S1 shows the clinical symptoms and signs, and underlying genetic defects. Two sex- and agematched healthy controls were recruited for each patient, and all subjects were enrolled to niacin supplementation (clinicaltrials.gov NCT03973203; primary end point, change in concentrations of $\mathrm{NAD}_{+}$and related metabolite levels in blood and muscle; for list of secondary endpoints see https://clinicaltrials.gov/ct2/show/record/NCT03973203?view=record]. Figure 1A and Figure S1 present the study design and procedures for selection of study subjects and data analyses, respectively. All the subjects were supplemented with a slowly increasing dose of niacin, from $250 \mathrm{mg} /$ day up to 750 or $1000 \mathrm{mg} / \mathrm{day}$ for four months, and we continued the follow-up of treatment effect up to 10 months in patients. These doses have been reported 
previously to elevate HDL cholesterol in patients with hypercholesterolemia (Vosper, 2009). Known niacin side-effects (hot flushes, tingling sensation of extremities) (Guyton and Bays, 2007) were experienced by all study subjects when the dose exceeded $500 \mathrm{mg} /$ day. These symptoms were, however, ameliorated upon continued use. The subjects also reported flatulence, gastrointestinal irritation and skin drying. One male patient reported transiently enhanced signs of gout. Two control subjects discontinued the study after two months due to gastrointestinal irritation; the remaining participants tolerated niacin well.

\section{PEO patients have disturbed NAD+ metabolism}

To elucidate baseline NAD+ metabolism, we quantified muscle NAD+ levels from the study subjects. Targeted liquid chromatography-mass spectrometry analysis of biopsy samples from vastus lateralis muscle demonstrated a $\sim 2$-fold decrease of muscle NAD+ concentrations in PEO patients compared to their matched controls (Fig. 1B). To understand the mechanism of muscle NAD+ depletion in our patients, we analyzed expression of genes encoding the main NAD+ biosynthetic pathway components (salvage and Preiss-Handler) and consumption enzymes in the muscle (Liu et al., 2018). Signs of impaired nicotinamide (NAM) utilization in the salvage pathway (downregulated mitochondrial NAM-nucleotide adenylyltransferase-3; $p<0.05$ ) and enhanced NAM elimination (trend of upregulated NAM $\mathrm{N}$-methyltransferase; $\mathrm{p}=0.064$ ) were found (Fig. S2A). In line with the latter finding, muscle NAM levels were significantly lowered in patients (Fig. S2B). Expression of poly(ADP-ribose) polymerase 1-2 isoforms, cluster-of-differentiation-38 and sirtuins was unaltered (Fig. $S 2 A, C)$. Overall, our data show that muscle $N A D_{+}$deficiency is characteristic for adult-onset mitochondrial myopathy.

We then asked whether the low muscle NAD+ concentration was also reflected systemically, as altered circulating NAD+ metabolome (Trammell and Brenner, 2013). Remarkably, patients had 2-fold lower blood NAD+ concentration than controls, with NADP 
concentration trending downwards, low NAM mononucleotide (NMN), but NAM riboside (NR) levels were similar (Fig. 1C-F). NAM and ADP-ribose, the metabolites produced by non-redox NAD+-dependent enzymes, were significantly higher than those of controls, suggesting increased $\mathrm{NAD}_{+}$consuming activities in patients (Fig. 1G-H). These results indicate that the primary muscle disease affected also systemic NAD+ levels, suggesting that blood NAD+ analysis has potential as a diagnostic and follow-up test for NAD+ booster therapy for mitochondrial myopathy.

\section{Niacin restores NAD+ concentrations in PEO patients}

Niacin supplementation increased muscle NAD+ content 1.3- and 2.3-fold in patients compared to baseline after four and 10 months, respectively, and in the later timepoint NAD+ levels had reached the healthy control values (Fig. 1B). In controls, muscle NAD+ content did not change, suggesting that steady-state NAD+ levels are tightly controlled in skeletal muscle (Fig. 1B). Niacin supplementation also remarkably elevated whole blood NAD+ concentrations both in patients (7.1-fold compared to baseline) and controls (5.7-fold) after four months (Fig. 1C). The blood NAD+ concentrations reached an 8.2-fold increase compared to baseline after 10 months of supplementation in patients (Fig. 1C). The concentrations of all NAD+ metabolites increased in the blood of both patients and controls (Fig. 1C-H). Given that niacin increased the levels of muscle and blood NAM (Fig. 1G, S2B) and ADP-ribose (Fig. 1H), niacin was likely metabolized as NAM via salvage pathway instead of Preiss-Handler pathway and it was effectively utilized by NAD+ consuming enzymes. The evidence indicates that niacin is a powerful NAD+ booster in humans, both healthy and diseased.

\section{Niacin improves body composition}


We next examined the impact of niacin on body composition. The baseline characteristics of the subjects are shown in Table S2. Niacin supplementation decreased whole-body fat percentage in controls and increased muscle mass both in controls and patients after 4-month supplementation, without a marked effect on intramyocellular lipid content or body weight (Fig. 2A-C, Fig. S3A). Strikingly, niacin supplementation reduced the patients' hepatic and visceral fat - the "unhealthy" fat depots increasing risk to metabolic syndrome - by $50 \%$ and $25 \%$, respectively, after 4 months of treatment (Fig. 2D-E). However, subcutaneous adipose tissue mass was unchanged in patients (Fig. 2F), indicating specificity in the regulatory role of $\mathrm{NAD}_{+}$and its derivatives in controlling fat deposits in mitochondrial disease. In healthy controls, with small overall fat depots, niacin did not significantly affect liver or visceral fat, but trended to decrease subcutaneous fat (Fig. 2D-F). Niacin has been previously observed to ameliorate diet-induced hepatic steatosis in rats (Ganji et al., 2014) and dyslipidemic humans (Hu et al., 2012). The decreasing fat-depots in our study subjects could be explained by increased oxidative metabolism (trend of increased energy expenditure after 4-month niacin supplementation (Fig. 2G); unchanged fasting free fatty acids despite of possible niacin-induced free fatty acid rebound (Guyton and Bays, 2007)) (Fig. S3B)). Food diaries showed no marked changes in eating habits or food consumption during supplementation (Fig. S3C-D), supporting the findings to be niacin-induced.

Niacin has been previously found to affect circulating levels of adiponectin (Plaisance et al., 2009; Westphal et al., 2007), an adipokine known to promote hepatic fatty acid oxidation (Giby and Ajith, 2014). We found the biologically active high-molecular weight form of adiponectin to be robustly increased in plasma in all niacin-treated subjects (Fig. $2 \mathrm{H}$ ) and to correlate negatively with liver fat content (Fig. 2l). These results suggest that NAD+ contributes to adiponectin signaling, the activation of which could explain the effects of niacin on liver fat content in mitochondrial disease patients. 


\section{Niacin effects on lipoproteins and erythropoiesis}

When entering the study, the patients showed lower levels of HDL and anti-atherogenic large HDL2b particles than controls (Fig. S4B-C). Niacin had no effect on total cholesterol but it increased HDL2b particles and decreased apolipoprotein B in controls and patients (Fig. S4A-D). Apolipoprotein B containing LDL and VLDL particles, the latter being reflected by circulating triglyceride levels, were reduced in patients after 10 months of niacin (Fig. S4E-F). No marked effects on other HDL subfractions, and apolipoprotein A or CIII levels were observed (data not shown). Of glucose metabolic effects, niacin increased fasting glucose levels both in controls and patients after 4-month supplementation, whereas insulin and Cpeptide were elevated only in controls at this time point (Fig. S4G-I). Glycosylated hemoglobin, reflecting long-term glucose levels, remained unchanged (the baseline glycosylated hemoglobin, insulin and C-peptide in the patients were higher than in controls, but remained within normal range except in one patient; Fig. S4H-J), as did liver function tests (Fig. S4K-L). These results indicate that niacin improved lipoprotein metabolism especially in mitochondrial myopathy patients.

We found niacin to slightly reduce hemoglobin concentration of patients after 4-month supplementation, but hemoglobin did not decrease beyond normal range during the study, except in one patient who showed anemia and iron deficiency already at baseline (Fig. S5A). Also, the number of erythrocytes decreased and thrombocytes trended downwards in patients, but leukocytes were unaffected (Fig. S5B-D). These results suggest that niacin treatment suppressed slightly erythropoiesis or iron metabolism. Folate and vitamin B12 are essential for erythropoiesis. Folate was lower in patients than controls at baseline, and it was unaffected by niacin treatment (Fig. S5E). Vitamin B12 decreased in patients during the first four months of niacin treatment - still remaining in normal range - but at 10-month time point returned to baseline (Fig. S5F). One patient with baseline vitamin-B12 deficiency was 
supplemented with vitamin B12 and iron (Fig. S5F). Our patients showed normal mean volumes of erythrocytes (Fig. S5G-H), excluding the possibility that the reduction in erythrocyte number was attributable to vitamin B12 deficiency. Unchanged plasma bilirubin concentration in all study subjects suggested that the reduced number of erythrocytes in patients was not explained by hemolysis, but was linked to mitochondrial disease physiology (Fig. S5I). These findings underscore the whole-organismal effects of niacin, and the necessity for follow-up of the patient blood counts when using NAD+ boosters, with special attention to hemoglobin concentration and blood counts.

\section{Niacin increases muscle strength}

Given that niacin as an NAD+ booster had remarkable metabolic effects, we explored whether it affected performance of the study subjects. After 10 months of niacin, patients showed improved muscle strength, differentially in different muscle groups: on the average 10-fold in abdominal muscles, 2-fold in back muscles, 2.5-fold in upper extremities (shoulder and elbow flexion strength), but little in lower extremities (knee extension strength 1.1-fold) as well as a slight improvement in the six-minute walking test (patients do not have ataxia, a symptom compromising six-minute walking test, and therefore the test results reflect walking performance) (Fig. 3A-F). Figure S6A-F shows the performance of the individual patients and Table S2 the baseline results of the six-minute walking test. Ocular muscle weakness, however, did not improve, which is consistent with severe atrophy of these early-affected muscles (Suomalainen et al., 1992) and the close-to-complete ophthalmoplegia of the patients. In controls, niacin improved muscle strength of upper (shoulder muscle strength) and lower (knee extension strength) extremities (Fig. 3D-F). In cardiopulmonary exercise testing, maximal oxygen uptake or maximal exercise capacity showed no significant changes after niacin supplementation (data not shown). However, the subjects reached quite variable 
maximal exercise capacity levels, which may have reflected their result as a group (data not shown). The levels of lactate, which were increased at baseline (Fig. 3G), decreased after exercise compared to baseline values in patients (Fig. $3 \mathrm{H}-\mathrm{I}$ ). The results suggest overall improvement of muscle metabolism in patients. Individual patients reported improved capacity to run or exercise, decreased frequency of muscle cramps and improved sleep. However, the questionnaires revealed that on the average, the muscle symptoms, quality of sleep, mood or the general quality of life of the subjects remained quite stable during niacin supplementation (Fig. S6G-N). These results indicate objective improvement of muscle strength in controls and patients after niacin supplementation.

\section{Niacin boosts mitochondrial biogenesis}

In mice, $\mathrm{NAD}_{+}$boosting improved morphological and molecular hallmarks of mitochondrial myopathy (Cerutti et al., 2014; Khan et al., 2014) when measured by histochemical in situ activities of the respiratory chain enzyme complexes cytochrome c oxidase (COX; respiratory chain complex IV, partially mtDNA-encoded) and succinate dehydrogenase (SDH; complex II, encoded by nuclear genes) in frozen muscle sections. In our patients, the number of COX-deficient, SDH-positive muscle fibers, as well as fibers with lowered complex I amount in vastus lateralis muscle decreased significantly after 10 months on niacin (Fig. 4A-B, Fig. S7A-B). Mitochondrial total COX activity and mass in muscle fibers increased both in controls and patients, indicating mitochondrial biogenesis induction (Fig. 4C-D; Fig. S7C-D). Table S2 shows the baseline results of COX activity and mitochondrial mass. However, the mtDNA deletion amount (Fig. S7E) or the ultrastructural abnormalities of mitochondria (distorted cristae, paracrystalline and electron-dense inclusions) were not corrected by niacin (Fig. 4E-F). These data indicate that morphological and genetic changes 
in mitochondria persisted in patients, but niacin induced mitochondrial biogenesis and respiratory chain activities in all study subjects.

Transmission electron microscopy indicated subsarcolemmal and intermyofibrillar glycogen accumulation in study subjects after niacin treatment (Fig. 4G-H), which was confirmed by glycogen staining on muscle sections (Fig. S7F). Muscle glycogen synthase (GYS1) expression was not elevated (patients: 0.93-fold increase at 10-month niacin;

controls: 0.95 -fold at 4-months), but the enzyme is also regulated by substrate and phosphorylation, and thereby we cannot exclude the induction of glycogen synthesis.

Of blood biomarkers of mitochondrial disease (Lehtonen et al., 2016; Suomalainen et al., 2011), serum fibroblast growth factor 21 (FGF21) or growth/differentiation factor 15 (GDF15) or their RNA levels in the skeletal muscle did not change after niacin treatment (Fig. S8A-B; Fig. 6E). However, niacin restored elevated plasma concentration of alanine, a biomarker for mitochondrial disorders, as well as plasma levels of the mitochondrially catabolized branched chain amino acids (valine and isoleucine) in patients (Fig. S8C). The plasma creatine kinase and myoglobin concentrations were unaffected, indicating no muscle damage in the study subjects (Fig. S8D-E), and muscle histology showed no signs of inflammatory cell infiltration. As a whole, NAD+ repletion restored blood amino acid levels, but had little effect on cytokine biomarkers of mitochondrial myopathy.

\section{Niacin regularizes $P E O$ muscle metabolism}

Considering the central role of $\mathrm{NAD}_{+}$and its derivatives as redox-regulators and gatekeepers to metabolic pathways, we performed targeted blood and muscle metabolomics analysis of 100 metabolites, covering well different biosynthetic pathways, in patients and controls. Unsupervised clustering of muscle metabolome revealed a clear shift of the global metabolite profile of patients towards controls after 10-months on niacin (Fig. 5A). Niacin and NAM metabolism was among the most significantly changed pathways, as a proof of 
principle, as were the biosynthetic pathways for purine, pyrimidine, methionine, and glycine metabolism, and bile acid and protein biosynthesis (Fig. 5B). PEO patient muscle has imbalanced nucleotide precursor amounts (Nikkanen et al., 2016), of which inosine monophosphate and adenosine of purine synthesis were brought back to control level by niacin treatment (Fig $5 C, E)$. NAD+ regulates conversion of hypoxanthine towards xanthine and allantoin, of which the two latter increased after niacin, and hypoxanthine decreased. Levels of pyrimidine nucleosides cytidine and deoxycytidine were also replenished by niacin in PEO muscle (Fig. 5C, E).

Niacin supplementation increased most amino acids in patients' muscle (Fig. 5D), whereas in the plasma they decreased (Fig. S8F), suggesting their increased synthesis/uptake in the muscle, instead of degradation of muscle protein. Muscle concentrations of creatine, an amino acid used for energy production, and its degradation product creatinine reached control levels after 10 months of niacin supplementation in patients (Fig. 5C, E). The increased availability of creatine drives the synthesis of high energy phosphates and recycling of ATP (Brosnan and Brosnan, 2007), which could contribute to the improved muscle performance of patients. Muscle strength could also be improved by significant increase of taurine levels (Fig. 5D-E), an amino acid essential for $\mathrm{Ca}_{2+-}$-dependent muscle excitation-contraction, antioxidant defense and cellular signaling (Spriet and Whitfield, 2015). The data suggest that niacin also diminished the utilization of S-adenosyl methionine, the primary methyl donor molecule, for polyamine biosynthesis: the high levels (up to 10 -fold) of spermidine and $5^{\prime}$-methylthioadenosine, a polyamine biosynthesis byproduct, in the patient muscle were reduced close to that of the controls' mean after niacin supplementation (Fig. 5C, E). These changes restore cellular methylation capacity, the largest user of which is muscle creatine synthesis (Mudd et al., 2007). Overall, our results 
highlight marked $\mathrm{NAD}_{+}$-dependent effects of 10 -month niacin for muscle nucleotide and amino acid metabolism and methyl cycle in mitochondrial myopathy.

\section{Niacin downregulates mTOR signaling pathways}

We then asked how niacin affected gene expression. The pathway enrichment analysis of RNA sequencing data indicated that serine/glycine/one-carbon pathways (de novo serine biosynthesis, purine degradation, glutathione metabolism) were among the most significantly changed in PEO (Fig. 6A; Table S3 shows individual transcripts). The finding is intriguing, as these pathways were also found to be modified in mitochondrial myopathy mice, as part of the mitochondrial integrated stress response (ISRmt) (Khan et al., 2017; Nikkanen et al., 2016; Tyynismaa et al., 2010), indicating high conservation of the stress responses in species. Compared to controls, PEO-muscle showed macrophage and innate immunity pathway activation ("phagosome formation"), less significant after niacin supplementation (Fig. 6A; Tables S3-5). Only five and two of the disease associated pathways remained significantly changed after 4- and 10-month supplementation, respectively, in patients as compared to controls (Tables S4-5). The most prominent niacin-related transcriptomic change in PEO patients' muscle (PEO 10-months on niacin vs PEO baseline) was widespread downregulation of mammalian target of rapamycin (mTOR)-dependent cytoplasmic translation, involving a large number of cytoplasmic ribosome subunits as well as translation initiation factor elF1 (Fig. 6B and Table S6). This was intriguing, as the major translation activator mTORC1 was also activated in mitochondrial myopathy mice, in COXnegative/SDH-positive fibers, and rapamycin (mTOR inhibitor) rescued their disease signs (Khan et al., 2017). In patients, niacin activated the peroxisome proliferator-activated receptor signaling pathway, known to induce mitochondrial biogenesis (Fig. 6B, Table S6), which we find to occur in our subjects' muscle. Niacin also led to decreased atherosclerosis signaling including lowered apolipoprotein expression in patients (Fig. 6B, Table S6). The healthy 
control muscles showed only few changes after 4-months of niacin (Fig. 6C, Table S7), emphasizing the importance of $\mathrm{NAD}_{+}$metabolic changes in mitochondrial myopathy.

To examine in detail the effects of NAD+ booster niacin in the mitochondrial integrated stress response (ISRmt), the main stress response in mitochondrial myopathy muscle in mice and human cell lines (Bao et al., 2016; Khan et al., 2017; Kuhl et al., 2017; Nikkanen et al., 2016), we studied the effects NAD+ boosting for the expression of 24 ISRmt target genes in patients' muscle. The analysis confirmed wide-spread induction of these genes, but their expression was unaffected by niacin (activating transcription factor 5 (ATF5) and its targets asparagine synthetase; tribbles pseudokinase 3, FGF21, GDF15, methylene-tetrahydrofolate dehydrogenase 2 and $1 \mathrm{~L}$; de novo synthesis of serine; phosphoserine aminotransferase 1 , phosphoglycerate dehydrogenase and serine hydroxymethyltransferase 2; glutathione metabolism; glutathione peroxidase 3 and glutathione reductase; and unfolded protein response endoplasmic reticulum; C/EBP homologous protein; Fig. 6D-G). Our data show that niacin serves as a metabolic "by-pass" therapy, providing functional benefits through suppression of mTOR signaling, cytoplasmic translation and metabolism, but without affecting the primary gene defects, disease-related stress signals or ISRmt. This finding indicates that it is possible to improve tissue metabolism and function without curing transcriptome or stress responses and emphasizes the importance of metabolomics as a readout of therapy effects, instead of the widely used transcriptomics.

\section{Discussion}

The revelation of disturbed NAD+ homeostasis in muscle and metabolic diseases (Gariani et al., 2016; Khan et al., 2014; Ryu et al., 2016; Trammell et al., 2016; Yoshino et al., 2011; Zhang et al., 2016) in animals has generated a high interest whether NAD+ metabolism aberrations occur in degenerative diseases in humans and whether progression of such 
disorders can be improved by NAD+ boosters (Rajman et al., 2018). NAD+ deficiency has been found to occur in congenital malformation syndromes or fatal febrile children's disorders caused by genetic defects in NAD+ metabolic enzymes (Shi et al., 2017; Van Bergen et al., 2019) and sarcopenia in aged humans (Migliavacca et al., 2019), but no data of secondary $N A D_{+}$deficiency caused by metabolic insult in other adult-onset degenerative disorders exist. Here, we show that mitochondrial muscle disease causes NAD+ deficiency, a myopathyinduced vitamin B3 deficiency, a metabolic pellagra. Furthermore, we show that NAD+ levels can be rescued by a potent $\mathrm{NAD}_{+}$booster niacin, a vitamin $\mathrm{B} 3$ form. We demonstrate that niacin remarkably restores muscle and systemic $\mathrm{NAD}_{+}$, and provides metabolic and functional benefits for patients with mitochondrial myopathy, indicating that NAD+ deficiency contributes to disease progression. In the healthy subjects, niacin did not increase muscle NAD+, despite the 5 -fold increase in the blood. These results suggest that in healthy muscle the NAD+ amounts are close to the homeostatic maximum. Our data implicate the potent effects of vitamin $\mathrm{B} 3$ forms on metabolism and present blood $\mathrm{NAD}_{+}$analysis as a powerful tool to identify patients and individuals with NAD deficiency. Metabolism of our different patients responded to niacin supplementation similarly, indicating that uniform disease manifestations share underlying molecular pathophysiology, despite different genetic background and disease duration. Future studies are needed to tell whether early treatment immediately after diagnosis might delay disease progression even further. Surprisingly, restoring NAD+ levels resulted in metabolic and functional benefits in patients, without considerable effects on mtDNA deletion load or mitochondrial ultrastructure, suggesting that "metabolic by-pass" of the primary cause of disease may have benefits. In mitochondrial myopathy mice, NAD+ boosting by NR (another vitamin B3 form) rescued both metabolism and disease signs (Cerutti et al., 2014; Khan et al., 2014). Whether the difference is explained by species-specific differences in muscle repair or 
turnover, or by distinct properties of the vitamin B3 forms as modifiers of mitochondrial metabolism, remains to be explored.

Our pilot study is a proof-of-principle of niacin effects on mitochondrial myopathy. Our data implicate niacin as a promising treatment for mitochondrial myopathy patients who show $N A D$ + deficiency, but effects for other patient groups are still unknown. Furthermore, the dose we chose for niacin supplementation was based on experience of its efficacy in hypercholesterolemia (Vosper, 2009), but the optimal dose for mitochondrial myopathy needs to be determined. As potential adverse effects that require attention and follow-up we report mildly decreasing hemoglobin concentration and erythrocyte counts, as well as increased muscle glycogen in the study subjects. Previously, NR treatment decreased hemoglobin and hematocrit in healthy subjects (Airhart et al., 2017). These findings suggest modifying functions of NAD+ boosters for iron metabolism or erythropoiesis and need for hemoglobin monitoring during vitamin B3 supplementation. The glycogen amounts can reflect storage or flux; the latter being supported by improved mitochondrial biogenesis and muscle strength of our patients. Glycogen flux has been reported to be simultaneous and separable from glucose utilization (Hardin and Kushmerick, 1994). However, as increased glycogen storage can have long-term harmful effects, it requires special attention in follow-up studies.

In conclusion, our data 1) underscore the potent role of micronutrient vitamin B3 as a metabolic modifier; 2) identify NAD+ deficiency as a contributor to mitochondrial myopathy progression; 3) point to usefulness of niacin therapy for PEO-patients; 4) introduce blood $N A D_{+}$test as a tool to identify and follow-up NAD+ deficiency; 5) indicate that correction of metabolome and function can occur without correction of transcriptional stress responses, emphasizing importance of metabolomic analysis in follow-up of treatment efficacy.

\section{Limitations of the study}


We report a pilot study of the effects of NAD+ booster niacin to muscle function and molecular physiology in mitochondrial myopathy patients and their matched healthy controls. The open study setting may compromise some results of patient performance and need to be followed up in a larger controlled trial. The study subjects volunteered to two to three thickneedle muscle samplings, yielding material sufficient for histologic and omics analyses, but not for representative western blot analyses of specific nutrient sensors or respirometry analyses. However, downstream targets of nutrient sensors and histochemical respiratory enzyme activities are reported. Whether blood NAD+ levels can be used more generally as a biomarker of disease, needs to be studied in future.

\section{Acknowledgements}

We thank the study participants for their valuable contribution to this research. We acknowledge Markus Innillä, Mia Urjansson, Katja Sohlo, Tarja Hallaranta, Tuula Manninen, Sari Räsänen, Helinä Perttunen-Nio and Minna Tuominen for their technical expertise. Jatin Nandania is acknowledged for the technical help in metabolomics sample analysis. CSC IT Center for Science, Finland, is acknowledged for providing the ePouta cloud computing environment for storage and processing of the data.

The study was supported by Centre of Excellence funding from the Academy of Finland (\#307431, AS and KP), as well as academy grants (307592 and 303349 (AS), 286359 (EP), 272376 (KHP), 314383 (KHP), 266286 (KHP)), Sigrid Jusélius Foundation, United Mitochondrial Disease Foundation, Finnish Medical Foundation, Novo Nordisk Foundation, Gyllenberg Foundation, Finnish Diabetes Research Foundation, Finnish Foundation for Cardiovascular Research, HiLife/University of Helsinki, Government Research Funds (Helsinki University Hospital). 


\section{Author Contributions}

E.P., M.A. and A.S. designed the study. E.P. and A.S. supervised the project and wrote the manuscript. E.P. and N.A.K. performed experiments and E.P. analyzed data. M.A. recruited and examined the study subjects and analyzed data. K.H.P., N.L., A.H., J.K., N.U., U.H.P.P. and K.H. did clinical examinations, sampling and/or did preliminary data analysis; M-RT contributed to analysis of blood lipid profiles. V.B. and N.A.K. analyzed transcriptomic data. A.P. and V.V. performed metabolomics analysis including data analyses. C.B. and M.S. analyzed NAD+ metabolome. All authors revised and approved the manuscript.

\section{Declaration of Interests}

A.S. has filed a patent application for a method that allows NAD+ analysis from the blood and tissue samples. C.B. is the inventor of intellectual property on the nutritional and therapeutic uses of NR. He serves as chief scientific advisor of ChromaDex, which licensed, developed, and commercialized NR technologies, and holds stock in ChromaDex. The other authors declare no competing interests.

\section{Main Figure Titles and Legends}

Figure 1. PEO patients show systemic and muscle NAD+ depletion which can be rescued by niacin.

(A) The schematic diagram of the study design. The daily niacin dose was gradually escalated from $250 \mathrm{mg} /$ day by $250 \mathrm{mg}$ per every four weeks to achieve the final treatment dose, $1 \mathrm{~g} /$ day. At the end of the study, the dose was decreased by $250 \mathrm{mg}$ per every four weeks. Clinical examinations and collection of muscle biopsies were performed in patients at the time points 0, 4 and 10 months and in controls at 0 and 4 months. Fasting blood samples 
were collected every second week until 4 months and thereafter every six weeks until the end of the study.

(B) Muscle NAD+ content before and after niacin supplementation in controls $(n=8)$ and patients $(n=5)$.

(C-H) Whole blood NAD+ metabolite levels; NAD+ (C), NADP (D), NAM mononucleotide (E), NAM riboside $(F)$, NAM $(G)$ and ADPR $(H)$ before and after niacin supplementation in controls $(n=8)$ and patients $(n=3-5)$.

Data are median \pm lowest/highest value. ${ }^{*} P<0.05 ;{ }^{*} P \leq 0.01 ;{ }^{* *} P \leq 0.001$. Baseline differences between controls and patients were analyzed using Mann-Whitney nonparametric test. Friedman non-parametric ANOVA was used to determine the effect of treatment on patients' values at different time points whereas control values before and after niacin supplementation were compared using Wilcoxon non-parametric test. See also Fig. S1 and Fig. S2. NMN, nicotinamide mononucleotide; NR, nicotinamide riboside; NAM, nicotinamide and ADPR, ADP ribose.

Figure 2. The effect of niacin on body composition, energy expenditure and different fat depots.

$(\mathrm{A}-\mathrm{H})$ Body weight $(\mathrm{A})$, whole body fat percentage $(\mathrm{B})$ muscle mass $(\mathrm{C})$, liver fat $(\mathrm{D})$, visceral fat $(E)$, subcutaneous fat $(F)$, energy expenditure $(G)$ and plasma high molecular weight (HMW), the biologically active form, of adiponectin $(H)$ compared to baseline in controls $(n=8)$ and patients $(n=5)$. Results are expressed as fold change (FC) compared to pretreatment stage.

(I) Correlation of liver fat percentage with plasma HMW adiponectin after 4-month niacin supplementation in all study subjects $(n=13)$. 
Data are median \pm lowest/highest value. Friedman non-parametric ANOVA was used to determine the effect of treatment on patients' values at different time points whereas control values before and after niacin supplementation were compared using Wilcoxon nonparametric test. Correlation between adiponectin levels and liver fat content was assessed with a Spearman nonparametric rank test. ${ }^{*} \mathrm{P}<0.05 ;{ }^{* *} \mathrm{P} \leq 0.01 ;{ }^{* *} \mathrm{P} \leq 0.001$. See also Table S2. and Fig. S3.

\section{Figure 3. Niacin improves muscle strength and performance.}

$(A-F)$ Distance travelled during 6 min walk test $(A)$, back muscle $(B)$, abdominal muscle $(C)$, repetitive shoulder muscle lift (D), elbow flexion $(E)$ and knee extension $(F)$ strength compared to baseline in controls $(n=8)$ and patients $(n=4-5)$. For isometric tests, the highest value from three repetitions was recorded. Results are expressed as fold change (FC) compared to pretreatment stage. One patient was excluded from the 6 min walk test due to a recent foot injury at 10-month time point.

(G-I) Lactate levels during resting, exercise and post-exercise recovery in a cardiopulmonary exercise test at baseline $(G)$ in controls $(n=8)$ and patients $(n=5)$, and pre and post niacin in controls $(H) n=8$ and patients $(I) n=5$.

Data are median \pm lowest/highest value. Friedman non-parametric ANOVA was used to determine the effect of treatment on patients' values at different time points whereas control values before and after niacin supplementation were compared using Wilcoxon nonparametric test. The baseline difference between groups and the effect of niacin on lactate levels in controls and patients were determined with two-way ANOVA with Dunnett's multiple comparison test. ${ }^{*} \mathrm{P}<0.05 ;{ }^{* *} \mathrm{P} \leq 0.01 ;{ }^{* *} \mathrm{P} \leq 0.001$. See also Table S2 and Fig. S6.

Figure 4. Niacin alleviates muscle histology and increases mitochondrial biogenesis. 
(A-B) Immunohistological assessment of cytochrome c oxidase (COX) negative/succinate dehydrogenase (SDH) positive (A) and complex I (Cl) negative (B) muscle fibers compared to baseline in patients $(n=4-5)$. Three and seven subunits of COX and $\mathrm{Cl}$ are encoded by mtDNA subunits, respectively, and thereby mtDNA deletions cause COX and $\mathrm{Cl}$-deficiency, whereas, complex II is completely nuclear-encoded, and not affected by mtDNA deletions. Results are expressed as fold change (FC) compared to pretreatment stage. Individual patient values are shown in small inserts. Samples from one patient were excluded from the assessment of COX negative/SDH positive muscle fibers due to poor sample quality.

(C-D) Immuhistochemical analysis of COX activity (C) and mitochondrial mass (D) compared to baseline in controls $(n=8)$ and patients $(n=5)$.

$(\mathrm{E}-\mathrm{F})$ Electron micrographs of subsarcolemmal mitochondria $(\mathrm{M})$ in one patient $(\mathrm{E})$ and one control $(F)$ at different time points. Scale bar, $1000 \mathrm{nM}$. Enlargements of the subsarcolemmal mitochondria are shown on the right-hand side for the patient sample at the time points 0 and 10 months.

(G-H) Electron micrograph image of one PEO patient $(G)$ and one control $(H)$ showing glycogen (G) deposition (marked with arrow) in muscle fiber (MF) after niacin. Scale bar, $1000 \mathrm{nM}$.

Results are expressed as fold change (FC) compared to pretreatment stage. Data are median \pm lowest/highest value. ${ }^{*} P<0.05 ;{ }^{*} P \leq 0.01$. Friedman non-parametric ANOVA was used to determine the effect of treatment on patients' values at different time points whereas control values before and after niacin supplementation were compared using Wilcoxon nonparametric test. See also Table S2, Fig. S7 and Fig. S8.

Figure 5. Niacin shifts muscle metabolite profile of patients towards controls. 
(A) Principal component analysis (PCA) of muscle metabolites in controls $(n=8)$ and patients $(n=5)$ at different time points.

(B) The most significantly changed metabolite pathways in patients $(n=5)$ upon 10-month niacin supplementation compared to baseline in muscle.

(C-D) The effect of niacin on muscle metabolites in patients $(n=5)$ as compared to controls $(n=8)$ in following pathways; purine, pyrimidine and polyamine metabolism $(C)$, creatine $(C)$, and amino acids (D). Data are median \pm lowest/highest value.

(E) One-carbon metabolism and associated pathways in patient muscle pre and post 10month niacin. Colored text, changed at baseline; red, increase and green: decrease. Circled metabolites, changed upon niacin; red, increase and green: decrease. SAH, S-adenosyl homocysteine; SAM, S-adenosyl methionine; IMP, inosine monophosphate; AMP, adenosine monophosphate; cAMP, cyclic adenosine monophosphate; GAA, guanidino-acetic acid; MTA, 5'methylthioadenosine; ROS, reactive oxygen species; THF, tetrahydrofolate, MTHFD, methylenetetrahydrofolate dehydrogenase; dTMP, deoxythymidine monophosphate. Baseline differences between controls and patients were analyzed using Mann-Whitney nonparametric test. Two-way ANOVA with Dunnett's multiple comparison test was used to determine the effect of treatment on patients' metabolite values at different time points. $\# p \leq$ $0.06,{ }^{*} P<0.05 ;{ }^{* *} P \leq 0.01 ;{ }^{* * *} P \leq 0.001$.

\section{Figure 6. The effect of niacin on muscle transcriptomic signatures.}

(A) Transcriptomic pathways changed in patients compared to control baseline based on Ingenuity Pathway Analysis analysis (controls $n=8$ and patients $n=4-5$ ).

(B-C) The effect of 10- (B) or 4-month (C) niacin on transcriptomic pathways in patients $(n=4$ 5) and controls ( $n=8)$ compared to baseline, respectively. 
(D-G) The effect of niacin on mitochondrial integrated stress response (ISRmt); activating transcription factor (ATFS) (D), and genes involved in ATF regulated ISRmt (E), serine biosynthesis $(F)$, and glutathione and unfolded protein response mitochondrial and endoplasmic reticulum $(G)(n=8)$ in patients $(n=4-5)$ compared to control baseline at time points 4 and 10 months. Results are expressed as fold change (FC) compared to pretreatment stage. Data are shown as mean \pm SEM. \#p $\leq 0.06,{ }^{*} P<0.05 ;{ }^{* *} P \leq 0.01 ;{ }^{* * *} P \leq$ 0.001. Statistical analyses are described under transcriptomics analysis section. GP6, glycoprotein 6; LXR, liver $X$ receptor; $R X R$, retinoid $X$ receptor; EIF, eukaryotic initiation factor; ILK, integrin-linked kinase; mTOR, mammalian target of rapamycin; PPAR, peroxisome proliferator-activated receptor; NO, nitric oxide; ROS, reactive oxygen species; PI, phosphatidylinositol; ATF, activating transcription factor; ASNS, asparagine synthetase; TRIB3, tribbles pseudokinase 3; FGF21, fibroblast growth factor 21; GDF15, growth/differentiation factor 15; MTHFD, methylene-tetrahydrofolate dehydrogenase; PSAT1, phosphoserine aminotransferase 1; PHGDH, phosphoglycerate dehydrogenase; SHMT, serine hydroxymethyltransferase; GXP3, glutathione peroxidase 3; GSR, glutathione reductase; CHOP, C/EBP homologous protein; LONP1, lon peptidase 1; CLPP, caseinolytic mitochondrial matrix peptidase; HSP, heat shock protein and mt, mitochondrial.

\section{STAR $\star$ Methods}

Detailed methods are provided in the online version of this paper and include the following:

- KEY RESOURCES TABLE

- RESOURCE AVAILABLILTY

- LEAD CONTACT

The Lead Contact is Professor Anu Suomalainen (anu.wartiovaara@helsinki.fi). 
- MATERIALS AVAILABILITY

This study did not generate new unique reagents. Requests for resources and reagents should be directed to the Lead Contact.

- DATA AND CODE AVAILABILITY

The RNA sequencing data are available in the Gene Expression Omnibus data repository with the accession code GSE129811.

- EXPERIMENTAL MODEL AND SUBJECT DETAILS

- Study participants

- METHODS DETAILS

- Subject recruitments and study design

- Body composition and energy expenditure

- Muscle strength and performance

- Blood laboratory examinations

- Muscle sampling and histology

- mtDNA analyses and quantification

- Targeted quantitative metabolomics analyses

- Transcriptomics analysis

- QUANTIFICATION AND STATISTICAL ANALYSIS

- Statistical Analysis

- ADDITIONAL RESOURCES 


\section{Experimental Model and Subject details}

\section{Study participants}

This study was approved by the ethics committee of the hospital district of Uusimaa and Helsinki (the protocol number 110/13/03/01/14) and the study was conducted according to the principles of the Declaration of Helsinki. The study was registered at clinicaltrials.gov entry NCT03973203. Written informed consent was obtained from all subjects. Five patients with mitochondrial myopathy (one male, four females) and ten healthy controls (two males, eight females) aged between 17 and 70 years participated our long-term niacin supplementation study (Table S1). None of them had participated treatment studies previously. One patient had cholesterol lowering medication (simvastatin, 10mg once per day) and in addition, two patients were supplemented with iron and/or vitamin B12 due to deficiency of these antianemic substances from the early stages of the trial. Detailed inclusion criteria were as follows: (i) manifestation of pure mitochondrial myopathy, with no major other symptoms or manifestations, caused by single or multiple deletions of mtDNA; (ii) agreed to avoid vitamin supplementation or nutritional products with vitamin B3 forms 14 days prior to the enrollment and during the study; (iii) written, informed consent to participate in the study; (iv) successful recruitment of age and gender matched healthy controls for each patient.

The exclusion criteria were: (i) inability to follow study protocol; (ii) pregnancy or breastfeeding at any time of the trial; (iii) malignancy that requires continuous treatment, (iv) unstable heart disease; (v) severe kidney disease requiring treatment; (vi) severe encephalopathy; (vii) regular usage of intoxicants; (viii) previous participation to intervention studies.

\section{Method Details}


Subject recruitments and study design

Patients and controls were recruited by national network of adult neurologist (treating patients with mitochondrial myopathy) and via our local community through advertisement, respectively. The procedures for the selection of study participants is described in Figure S1. One-to-one phone conversations were conducted at least once a month to follow up compliance to the study and potential symptoms or side-effects. All participants were instructed to continue their normal routine and not make any changes to their physical activity and diet.

The examination protocol of this non-randomized, open-label study is described in Figure $1 \mathrm{~A}$. The niacin dose, $1 \mathrm{~g} / \mathrm{day}$, was selected on the basis of previous studies showing a favourable effect on lipid profiles in humans (Bruckert et al., 2010). To avoid the well-known side-effect of niacin, cutaneous flushing, controls and patients were supplemented with slowreleased form of niacin. The niacin dose was gradually escalated from $250 \mathrm{mg} / \mathrm{day}$ up to 1 g/day (Fig. 1A), except for the lightest study subject up to $750 \mathrm{mg} /$ day. The final dose was calculated based on body weight to be between 14 to $18 \mathrm{mg} / \mathrm{kg}$. The total intervention time was 4 and 10 months for controls and patients, respectively. Study subjects were advised to take niacin orally with a light meal in the evening.

\section{Body composition and energy expenditure}

Magnetic resonance imaging (MRI)/ magnetic resonance spectroscopy (MRS) experiments were performed on a 3.0 Tesla clinical imager (Verio, Siemens, Erlangen, Germany). Point resolved spectroscopy (PRESS) sequence was used for volume selection in hepatic and muscle MRS. For determination of intramyocellular lipids, a 6x6x20 mm3 MRS voxel was placed in the soleus muscle and both water-suppressed (48 averages) and 
unsuppressed (4 averages) spectra were obtained with time of repetition (TR) of $4000 \mathrm{~ms}$ and echo time (TE) of $30 \mathrm{~ms}$. Signal intensities from intramyocellular lipids were determined from water-suppressed spectra with LCModel v6.3 software using unsuppressed water signal as an internal reference. In hepatic MRS, a 25x25x25 mm3 voxel was placed in the middle of the right liver lobe and liver spectra with TE of $30 \mathrm{~ms}$ and four averages were collected. Signal acquisition was triggered to end exhalation using navigator belt to eliminate motion artifacts due to respiratory motion so that TR was kept $>4000$ ms. Liver spectra were analyzed with jMRUI 6.0 software (Stefan et al., 2009) and intensities of methylene and water resonances were determined using the AMARES algorithm (Vanhamme et al., 1997). Signal intensities were corrected for relaxation effects and liver fat determined as an intensity ratio of methylene/(methylene+water). Ratios were further converted to mass fractions as described previously (Kotronen et al., 2009). Briefly, since signal intensities are proportional to the number of resonating protons inside the voxel, the following experimentally determined factors, which have been previously used and validated by Longo et al. (Longo et al., 1995) and Szczepaniak et al. (Szczepaniak et al., 1999,) could be used to convert intensity ratios into weight fractions: (1) the ratio of the number of lipid protons in the fitted $(\mathrm{CH} 2) n-2$ signal to the total number of lipid protons is 0.633230 ; (2) proton densities of fat and water are 111 and $111 \mathrm{~mol} / \mathrm{L}$, respectively; (3) $1 \mathrm{~g}$ liver tissue contains $711 \mathrm{mg}$ water; (4) densities of the liver tissue, fat in the liver, and water are $1.051 \mathrm{~g} / \mathrm{mL}, 0.900 \mathrm{~g} / \mathrm{mL}$, and $1.000 \mathrm{~g} / \mathrm{mL}$; respectively.

A stack of abdominal T1-weighted MR images (16 slices, slice thickness $10 \mathrm{~mm}$, TR of $91 \mathrm{~ms}$, TE of $5.2 \mathrm{~ms}$ and flip angle of $80^{\circ}$ ) were obtained from $8 \mathrm{~cm}$ above to $8 \mathrm{~cm}$ below the $L 4 / 5$ lumbar intervertebral disks using frequency selective fat excitation. Areas of visceral and subcutaneous adipose tissue depots were determined from each slice using SliceOmatic (TomoVision, Quebec, Canada) 5.0 segmentation software utilizing region growing routine. 
Whole body fat percentage, muscle mass and energy expenditure were measured via bioelectrical impedance analysis using Tanita MC-980 device.

\section{Muscle strength and performance}

Study subjects' exercise capacity and muscle strength were tested via 6-min walk test, static strength of back muscles, and dynamic strength of abdominal and shoulder muscles. In addition, isometric muscle strength including elbow flexion and extension and knee extension was analyzed using Good strength Metitur adjustable dynamometer chair (Metitur Oy, Finland) (Era et al., 1992). Muscle symptoms were assessed via muscle questionnaire, focusing on the main symptoms of PEO patients such as muscle pain, numbness, tiredness, weakness and cramps in different muscle groups. The general quality of life was determined via RAND-36 questionnaire. The quality of sleep and depression was evaluated via the Basic Nordic Sleep and the Beck Depression Inventory questionnaires, respectively. Cardiopulmonary exercise test was performed on a bicycle ergometer with the initial workload of 30-40 W for patients, $40 \mathrm{~W}$ for control women, and $50 \mathrm{~W}$ for control men (Ollila et al., 2017). The work load was then increased by 40 or $50 \mathrm{~W}$, respectively, at 3-min intervals until level 17-19/20 on the Borg scale for perceived exertion was reached and respiratory quotient $\mathrm{RQ}\left(=\mathrm{VCO} / \mathrm{VO}_{2}\right)>1 \cdot 0$. Blood samples were collected for lactate measurement during rest, light exercise and maximum exercise, and $2 \mathrm{~min}, 4 \mathrm{~min}, 6 \mathrm{~min}, 10 \mathrm{~min}, 20 \mathrm{~min}, 30 \mathrm{~min}$ and 40 min post-exercise. Lactate levels were analyzed using standardized methods at the HUSLAB laboratories.

\section{Blood laboratory examinations}

Blood samples were collected after overnight fasting. Whole blood, and separated plasma and serum samples were frozen at $-80^{\circ} \mathrm{C}$. Blood count, alanine aminotransferase, 
asparate aminotransferase, creatine kinase, myoglobin, glucose, insulin, c-peptide, glycosylated hemoglobin, total cholesterol, LDL and triglycerides were analyzed using standardized methods at the HUSLAB laboratories. Plasma HDL was measured as cholesterol after precipitation of other lipoproteins with heparin-Mn2+ (Bachorik and Albers, 1986). HDL subspecies including HDL2b were determined with native gradient gel electrophoresis (Blanche et al., 1981). Gels were scanned with Epson scanner and analyzed with ImageQuant TL software. For each subspecies, the relative area under the densitometric scan curve was reported. Free fatty acids were determined using Wako Chemicals NEFA kit while apolipoprotein B levels were assessed with ApoB Konelab kit. FGF21 was analyzed using BioVendor human FGF21 ELISA kit, whereas serum GDF15 and plasma HMW adiponectin levels were measured using ELISA kits from R\&D systems.

\section{Muscle sampling and histology}

A Bergström needle biopsy from vastus lateralis was collected from controls at 0 and 4 months and from patients at the time points 0,4 and 10 months (Fig. 1A). The muscle sample for RNA, DNA and metabolite analyses were snap frozen in liquid nitrogen and samples for muscle histology were snap frozen in liquid nitrogen cooled isopentane. Twelve micrometer thick frozen muscle sections were used to measure cytochrome c oxidase (COX) and succinate dehydrogenase (SDH) activities using standard immunohistochemical activity assays (Forsstrom et al., 2019). Buffer composition used for the assay were; COX: $0.05 \mathrm{M}$ phosphate buffer ( $\mathrm{pH}$ 7.4) with 3,3-diaminobenzidine (DAB), catalase, cytochrome $\mathrm{c}$ and sucrose and for with incubation time of 60 minutes (RT) and for SDH: $0.05 \mathrm{M}$ phosphate buffer ( $\mathrm{pH}$ 7.4) with nitro-blue tetrazolium and sodium succinate; incubation time 90 minutes for $\operatorname{SDH}\left(+37^{\circ} \mathrm{C}\right)$. Frozen muscle sections were also stained for $\mathrm{NADH}$ :ubiquinone oxidoreductase 1 subunit B4, complex I subunit, and mitochondria, by standard 
immunohistochemical procedures, as published previously (Ahola et al., 2016). After stainings the slices were dehydrated in ascending alcohols, xylene-treated and mounted. Details about the key chemicals and antibodies used are listed in Key resource table. Approximately $170-1050$ cells per subject were counted to assess COX-negative/SDHpositive muscle fibers and Cl-negative muscle fibers at each time point of sample collection. For total COX activity and mitochondria mass 50-100 muscle fibers for each subject were quantified using image $\mathrm{J}$ software. Glycogen content in muscle was assessed with standard Periodic acid-Schiff staining from 8 um paraffin fixed muscle sections according to wellestablished protocols.

For transmission electron microscopy (TEM) analysis muscle samples were fixed in $2.5 \%$ glutaraldehyde. For plastic embedding they were then treated with $1 \%$ osmium tetroxide dehydrated in ethanol and embedded in epoxy resin. One $\mathrm{mm}$ section was used for stained with ethyl blue $(0.5 \% \mathrm{w} / \mathrm{v})$ and boric acid $(1 \% \mathrm{w} / \mathrm{v})$. The methyl blue sections were used to mark the interesting areas for the ultrastructural analyses by analyzing the sections with light microscope. For further analysis with transmission electron microscopy, ultrathin (60-90 nm) sections were cut on the grids and stained with uranyl acetate and lead citrate and viewed with JEOL 1400 Transmission Electron Microscopy (Ahola et al., 2016).

$m t D N A$ analyses and quantification

DNA was extracted from the snap frozen muscle samples using standard phenolchloroform extraction and ethanol precipitation method. MtDNA deletion analysis was performed by long-range PCR by amplifying a $8.3 \mathrm{~kb}$ region of mtDNA from nucleotide position 8232-16496 using Expand Long Template Enzyme and $10 \mathrm{ng}$ total DNA from each individual. The PCR amplification was done with an annealing temperature of $63^{\circ} \mathrm{C}$, with the extension times of $30 \mathrm{~s}$ or $3 \mathrm{~min}$. The short extension time efficiently amplifies short, deletion- 
containing mtDNA fragments (Hakonen et al., 2007). The mtDNA deletions were semiquantified by densitometry using a short mtDNA fragment amplified from cytb gene, after electrophoresis on $1 \%$ agarose gel and image-analysis using ChemiDoc ${ }^{\mathrm{TM}} \mathrm{XRS}+$ System (Bio-Rad). Cytb gene was amplified using Phusion high-fidelity DNA polymerase and GC buffer: initial denaturation of $30 \mathrm{~s}$ at $98^{\circ} \mathrm{C} ; 20$ cycles of $10 \mathrm{~s}$ at $98^{\circ} \mathrm{C}$ and 3 min at $72^{\circ} \mathrm{C}$; final extension step of $10 \mathrm{~min}$ at $72^{\circ} \mathrm{C}$. The PCR primers are provided in key resource table.

\section{Targeted quantitative metabolomics analyses}

The measurement of complete whole blood NAD+ metabolome was carried out after dual extractions as recently described (Trammell and Brenner, 2013; Trammell et al., 2016a). Briefly, for analysis of NR, NAM, niacin, MeNAM, Me2PY and Me4PY (group A analytes), samples were spiked with $400 \mathrm{pmol}$ of [1801]-NAM and [d4]- niacin, as well as $200 \mathrm{pmol}$ of [18O1]-NR, [d3]-Me4PY, and [d3, 18O1]-MeNAM (internal standard A). For analysis of NAD,, NADP, NMN, NAR, NAAD and ADPR, samples were dosed with 13C-yeast extract (internal standard B) as described (Trammell and Brenner, 2013; Elhassan et al., 2019).

To quantify NR, NAM, niacin, MeNAM, NAM oxide, Me2PY and Me4PY (group A analytes), $75 \mu \mathrm{l}$ of whole blood was added to $20 \mu \mathrm{L}$ group A internal standard and $500 \mu \mathrm{l}$ of 3:1 4\% trichloroacetic acid (TCA):acetonitrile was added. The mixture was allowed to sit on ice for $20 \mathrm{~min}$, after which samples were sonicated twice for 20 seconds and centrifuged at $+4^{\circ} \mathrm{C}$ for 13 minutes at $16.1 \mathrm{rcf}$. Next supernatant was removed and dried under vacuum overnight at room temperature. The samples were reconstituted in $2 \%$ acetonitrile/water immediately prior to the analytical run. To quantify NAD+, NADP, NMN, NAR, NAAD and ADPR (group B analytes), $75 \mu \mathrm{l}$ of whole blood was added to $20 \mu \mathrm{L}$ group B internal standard prepared in water and mixed with $500 \mu \mathrm{l}$ of 3:1 4\% (TCA):acetonitrile with vortexing. After resting on ice, the samples were centrifuged as described above. Next supernatant was 
removed and dried under vacuum overnight, and reconstituted in $2 \%$ acetonitrile/water. After reconstitution, samples were transferred to Waters polypropylene plastic total recovery vials and stored in a Waters Acquity $\mathrm{H}$ class autosampler maintained at $+8^{\circ} \mathrm{C}$ until injection. In all cases, $8 \mu$ l of extract was loaded onto the column. For one set of samples, group B analytes plus Me4PY were extracted as for the group $B$ analytes. One hundred $\mu \mathrm{L}$ of blood was used and 200 pmol d3-Me4PY was added to the internal standard mix. All analytes in this group except NAAD have a corresponding stable labeled internal standard. Labelled NAD+ was used as the internal standard for NAAD. In group A Nam oxide used $18 \mathrm{O}-\mathrm{NAM}$ as its internal standard and Me-2-py used d3-Me-4-py as internal standard.

Separation and quantitation of analytes were performed with a Waters Acquity LC interfaced with a Waters TQD mass spectrometer operated in positive ion multiple reaction monitoring mode as described (Trammell and Brenner, 2013) with minor modifications. MRM transitions monitored were: $\mathrm{NAD}_{+} 664.1>136.1 ;{ }_{13} \mathrm{C}_{10}-\mathrm{NAD}+674.1>136.1 ; \quad \mathrm{NADP}$ 744.1>136.1; ${ }_{13} \mathrm{C}_{10}$-NADP $754.1>136.1$; NAAD 665.1>136.1; NMN 335>123: ${ }_{13} \mathrm{C}_{5}-\mathrm{NMN}$ 340>123; NAR 256>124; 13 C5-NAR 261>124; ADPR 560>136.1; $13 \mathrm{C}_{10}$-ADPR 570>136.1; NR 255>123; $18 \mathrm{O}-\mathrm{NR}$ 257>125; NAM 123>80; $18 \mathrm{O}-\mathrm{NAM}$ 125>80; MeNAM 137>94; 18O,d 3 MeNAM 142>97; NA 124>80; d4-NA 128>84; Me-4-py 153>136; d3-Me-4-py 156>139; Me2-py 153>110; NAM oxide 139>106. Separate Hypercarb columns (Thermo Scientific) were used for the separations. The conditions for group A were solvent $\mathrm{A} 10 \mathrm{mM} \mathrm{NH} 4 \mathrm{OAc}$ with $0.1 \%$ formic acid, solvent $B$ acetonitrile with $0.1 \%$ formic acid, solvent $D$ methanol, flow 0.30 $\mathrm{mL} / \mathrm{min}$; gradient initial 98\% A, 2\% B; 2.25 min, 98\% A 2\% B; 11 min, 74.8\% A 17.9\% B 7.3\% D; $11.1 \mathrm{~min}, 10 \%$ A 90\% B; $14.3 \min 10 \%$ A 90\% B; $14.4 \mathrm{~min}, 98.2 \%$ A 2\% B; end $18.5 \mathrm{~min}$. Conditions for Group B were solvent A $7.5 \mathrm{mM} \mathrm{NH}_{4} \mathrm{OAc}$ with $0.05 \% \mathrm{NH}_{4} \mathrm{OH}$, solvent $B$ acetonitrile with $0.05 \% \mathrm{NH} 4 \mathrm{OH}$; flow $0.353 \mathrm{~mL} / \mathrm{min}$; gradient initial $97 \% \mathrm{~A} 3 \% \mathrm{~B} ; 1.8 \mathrm{~min}$, 
$97 \%$ A 3\% B; $10 \min 65.5 \%$ A 34.5\% B; 11 min 10\% A 90\% B; 13.2 min, 10\% A 90\% B; 13.3 $\min , 97 \%$ A $3 \%$ B; end 19 min.

Targeted metabolomic analysis of 100 metabolites was performed from muscle and plasma from each subject in the study group using Water Acquity ultra performance liquid chromatography (UPLC) and triple-quadrupole mass spectrometry analysis. The metabolite extraction protocol is described and validated in detail in (Nandania et al., 2018a; Nandania et al., 2018b). Approximately $20 \mathrm{mg}$ of snap frozen muscle tissue was homogenized in Precellys homogenizing tubes containing $1.4 \mathrm{~mm}$ ceramic (zirconium oxide) beads with 20 $\mu \mathrm{L}$ of labeled internal standard mix and $500 \mu \mathrm{L}$ of extraction solvent [100\% acetonitrile (ACN), $1 \%$ formic acid (FA)] as extraction solvent using Precellys-24 homogenizer (Bertin Technologies, France) with following protocol; three cycles, $20 \mathrm{~s}$ each, at 5,500 rpm; $30 \mathrm{~s}$ pause between each homogenization interval (Khan et al., 2017). After homogenization, the samples were centrifuged for $10 \mathrm{~min}, 5000 \mathrm{rpm}$, at $+4^{\circ} \mathrm{C}$ in an Eppendorf $5404 \mathrm{R}$ centrifuge and the supernatant was collected. Same homogenization cycles were repeated after adding $500 \mu \mathrm{L}$ of $90 / 10 \% \mathrm{ACN} / \mathrm{H}_{2} \mathrm{O}+1 \% \mathrm{FA}$ to the remaining pellet in homogenization tubes, supernatants were pooled and filtered to OstroTM plate by applying vacuum at delta pressure of 300 psi for 3-5 min. The clean extract was collected to a 96 -well plate and was centrifuged for $15 \mathrm{~min}, 4000 \mathrm{rpm},+4^{\circ} \mathrm{C}$ and placed in auto-sampler of the liquid chromatography system for the injection.

For plasma samples, $100 \mu \mathrm{L}$ of plasma samples was mixed to $10 \mu \mathrm{L}$ of labeled internal standard mixture (Nikkanen et al., 2016). Metabolites were extracted with 1:4 (sample:solvent) of $100 \% \mathrm{ACN}+1 \% \mathrm{FA}$ solvent and the extracts were dispensed to OstroTM 96-well plate (Waters Corporation, Milford, USA). The collected extracts were dispensed in and filtered by applying vacuum at a delta pressure of 300-400 mbar for $2.5 \mathrm{~min}$ on robot's vacuum station. The clean extract was collected to a 96 -well collection plate, placed under 
OstroTM plate. The collection plate was sealed and centrifuged for $15 \mathrm{~min}, 5000 \mathrm{rpm},+4^{\circ} \mathrm{C}$ and placed in auto-sampler of the liquid chromatography system for the injection. Samples were analyzed on an ACQUITY UPLC-MS/MS system (Waters Corporation, Milford, MA, USA) with specific settings (Nandania et al., 2018a; Nandania et al., 2018b). Detection was performed on a Xevo TQ-S tandem triple quadrupole mass spectrometer (Waters, Milford, MA, USA), operated in both positive and negative polarities. Samples were ionized by electro spray ionization (ESI) and dwell time and subsequent data acquisition were automatically calculated by MassLynx 4.1 software and processed using TargetLynx software. Quantification was performed by internal standards and external calibration curves. The unsupervised principal component analysis (PCA) was used to study separation between the groups and treatments. The metabolite pathway analysis was done using MetaboAnalyst (Chong et al., 2018; Goeman and Buhlmann, 2007).

\section{Transcriptomics analysis}

Total muscle RNA was extracted with standard TRIzol and chloroform method and purified with RNA purification kit (RNeasy; Qiagen). A total of $1 \mathrm{ug}$ of total RNA from five PEO patients at three time points of treatment and eight controls with two time points was used for global transcriptomics analysis by RNA sequencing which was performed by the Beijing Genomic Institute (BGI) using their standard protocols. For pathway analysis, the 500 most changed transcripts (log2 fold changes) were selected regardless of P-value and subjected to pathway analysis using Ingenuity Pathway Analysis (IPA).

Data analysis was performed as follows. High-throughput RNA sequencing short reads were obtained for 8 healthy control at all time points under a 50 bp single-end read strategy. In contrast, short reads were obtained for 13 out of 15 patient samples as sequencing of one patient sample both at time point 0 month and 10 month failed. The 
resulting raw reads were preprocessed by the sequencing provider to filter out reads failing criteria for quality, unknown base and adaptor sequence content. Clean reads, specified in FASTQ format, amounted to approximately $26 \mathrm{M}$ per sample. The reads were aligned against the GRCh38 Human genome assembly - DNA primary assembly in FASTA format and gene annotation in GTF format from Ensembl release 92 - using STAR 2.5.0a (Dobin et al., 2013), giving read alignments in BAM format. Counting of reads mapping to genes was performed with htseq-count, HTSeq 0.10.0 (Anders et al., 2015), in union overlap resolution mode. The obtained read counts were then used for differential gene expression analysis with the Bioconductor DESeq2 1.18.1 package (Love et al., 2014). The differential expression analysis function in the package performs estimation of dispersion values across samples for each gene, estimation of size factors for normalization of differences in sequencing depth between the samples, and fitting of a negative binomial generalized linear model using both size factors and dispersion values to give estimates of fold change in gene expression. Fold changes in binary logarithmic scale and respective $p$-values were extracted for pairwise comparisons between a total of 5 experiment groups, namely, control individuals at baseline and at treatment time point 4 months, and patients at baseline and at treatment time points 4 and 10 months. When comparing control groups, e.g. controls at treatment time point 4 months to controls at baseline, or patient groups, e.g. patients at treatment time point 10 months to patients at time point 4 months, the group-specific effect of treatment was estimated controlling for individual effects.

\section{Quantification and statistical analysis}

Statistical Analysis 
Statistical analyses were performed in GraphPad Prism version 6.00 for Mac. Due to low number of study subjects, non-parametric tests were used without data normality assessment. Baseline differences between controls and patients were analyzed using MannWhitney non-parametric test. Friedman test with Dunn's multiple comparison test, a non-parametric equivalent of ANOVA with repeated measures, was used to determine the effect of treatment on patients' values at different time points whereas control values before and after niacin supplementation were compared using Wilcoxon matched-pairs signedranked non-parametric test. Correlation between adiponectin levels and liver fat content was assessed with a Spearman nonparametric rank test. The effect of niacin on multiple parameters such as plasma and muscle metabolites or plasma lactate levels at different times points was evaluated with two-way ANOVA with Dunnett's multiple comparison test both in controls and patients. Data are expressed as median \pm lowest/highest value and a $P$ value 0.05 was used as a threshold to mark a statistically significant result. The statistics can also be found in the figures and legends.

\section{Additional resources}

Clinicaltrials.gov identifier NCT03973203

\section{References}

Ahola, S., Auranen, M., Isohanni, P., Niemisalo, S., Urho, N., Buzkova, J., Velagapudi, V., Lundbom, N., Hakkarainen, A., Muurinen, T., et al. (2016). Modified Atkins diet induces subacute selective ragged-red-fiber lysis in mitochondrial myopathy patients. EMBO Mol. Med. 8, 1234-1247.

Airhart, S.E., Shireman, L.M., Risler, L.J., Anderson, G.D., Nagana Gowda, G.A., Raftery, D., Tian, R., Shen, D.D., and O'Brien, K.D. (2017). An open-label, non-randomized study of the 
pharmacokinetics of the nutritional supplement nicotinamide riboside (NR) and its effects on blood NAD+ levels in healthy volunteers. PloS One 12, e0186459.

Anders, S., Pyl, P.T., and Huber, W. (2015). HTSeq--a Python framework to work with highthroughput sequencing data. Bioinformatics 31, 166-169.

Bao, X.R., Ong, S.E., Goldberger, O., Peng, J., Sharma, R., Thompson, D.A., Vafai, S.B., Cox, A.G., Marutani, E., Ichinose, F., et al. (2016). Mitochondrial dysfunction remodels onecarbon metabolism in human cells. Elife 5, e10575.

Belenky, P., Bogan, K.L., and Brenner, C. (2007a). NAD+ metabolism in health and disease. Trends Biochem. Sci. 32, 12-19.

Belenky, P., Racette, F.G., Bogan, K.L., McClure, J.M., Smith, J.S., and Brenner, C. (2007b). Nicotinamide riboside promotes Sir2 silencing and extends lifespan via Nrk and Urh1/Pnp1/Meu1 pathways to NAD+. Cell 129, 473-484.

Bieganowski, P., and Brenner, C. (2004). Discoveries of nicotinamide riboside as a nutrient and conserved NRK genes establish a Preiss-Handler independent route to NAD+ in fungi and humans. Cell 117, 495-502.

Blanche, P.J., Gong, E.L., Forte, T.M., and Nichols, A.V. (1981). Characterization of human high-density lipoproteins by gradient gel electrophoresis. Biochim. Biophys. Acta 665, 408419.

Brosnan, J.T., and Brosnan, M.E. (2007). Creatine: endogenous metabolite, dietary, and therapeutic supplement. Annu. Rev. Nutr. 27, 241-261.

Bruckert, E., Labreuche, J., and Amarenco, P. (2010). Meta-analysis of the effect of nicotinic acid alone or in combination on cardiovascular events and atherosclerosis. Atherosclerosis 210, 353-361.

Canto, C., Menzies, K.J., and Auwerx, J. (2015). NAD+ Metabolism and the Control of Energy Homeostasis: A Balancing Act between Mitochondria and the Nucleus. Cell Metab. 22, 3153.

Cerutti, R., Pirinen, E., Lamperti, C., Marchet, S., Sauve, A.A., Li, W., Leoni, V., Schon, E.A., Dantzer, F., Auwerx, J., et al. (2014). NAD-Dependent Activation of Sirt1 Corrects the Phenotype in a Mouse Model of Mitochondrial Disease. Cell Metab. 19, 1042-1049.

Dobin, A., Davis, C.A., Schlesinger, F., Drenkow, J., Zaleski, C., Jha, S., Batut, P., Chaisson, M., and Gingeras, T.R. (2013). STAR: ultrafast universal RNA-seq aligner. Bioinformatics 29, 15-21.

Elhassan, Y.S., Kluckova, K., Fletcher, R.S., Schmidt, M.S., Garten, A., Doig, C.L., Cartwright, D.M., Oakey, L., Burley, C.V., Jenkinson, N., et al. (2019). Nicotinamide Riboside 
Augments the Aged Human Skeletal Muscle NAD+ Metabolome and Induces Transcriptomic and Anti-inflammatory Signatures. Cell Rep. 28, 1717-1728 e1716.

Era, P., Lyyra, A.L., Viitasalo, J.T., and Heikkinen, E. (1992). Determinants of isometric muscle strength in men of different ages. Eur. J. Appl. Physiol. Occup. Physiol. 64, 84-91.

Försstrom, S., Jackson, C.B., Carroll, C.J., Kuronen, M., Pirinen, E., Pradhan, S., Marmyleva, A., Auranen, M., Kleine, I.M., Khan, N.A., et al. (2019). Fibroblast Growth Factor 21 Drives Dynamics of Local and Systemic Stress Responses in Mitochondrial Myopathy with mtDNA Deletions. Cell Metab. 30, 1040-1054 e1047.

Ganji, S.H., Kukes, G.D., Lambrecht, N., Kashyap, M.L., and Kamanna, V.S. (2014). Therapeutic role of niacin in the prevention and regression of hepatic steatosis in rat model of nonalcoholic fatty liver disease. Am. J. Physiol. Gastrointest. Liver Physiol. 306, G320327.

Gariani, K., Menzies, K.J., Ryu, D., Wegner, C.J., Wang, X., Ropelle, E.R., Moullan, N., Zhang, H., Perino, A., Lemos, V., et al. (2016). Eliciting the mitochondrial unfolded protein response by nicotinamide adenine dinucleotide repletion reverses fatty liver disease in mice. Hepatology 63, 1190-1204.

Giby, V.G., and Ajith, T.A. (2014). Role of adipokines and peroxisome proliferator-activated receptors in nonalcoholic fatty liver disease. World J. Hepatol. 6, 570-579.

Guyton, J.R., and Bays, H.E. (2007). Safety considerations with niacin therapy. Am. J. Cardiol. 99, 22C-31C.

Hardin, C.D., and Kushmerick, M.J. (1994). Simultaneous and separable flux of pathways for glucose and glycogen utilization studied by 13C-NMR. J. Mol. Cell Cardiol. 26, 1197-1210. Holt, I.J., Cooper, J.M., Morgan-Hughes, J.A., and Harding, A.E. (1988). Deletions of muscle mitochondrial DNA. Lancet 1, 1462.

Hu, M., Chu, W.C., Yamashita, S., Yeung, D.K., Shi, L., Wang, D., Masuda, D., Yang, Y., and Tomlinson, B. (2012). Liver fat reduction with niacin is influenced by DGAT-2 polymorphisms in hypertriglyceridemic patients. J. Lipid Res. 53, 802-809.

Katsyuba, E., Mottis, A., Zietak, M., De Franco, F., van der Velpen, V., Gariani, K., Ryu, D., Cialabrini, L., Matilainen, O., Liscio, P., et al. (2018). De novo NAD+ synthesis enhances mitochondrial function and improves health. Nature 563, 354-359.

Kaukonen, J., Juselius, J.K., Tiranti, V., Kyttala, A., Zeviani, M., Comi, G.P., Keränen, S., Peltonen, L., and Suomalainen, A. (2000). Role of adenine nucleotide translocator 1 in mtDNA maintenance. Science 289, 782-785. 
Khan, N.A., Auranen, M., Paetau, I., Pirinen, E., Euro, L., Försstrom, S., Pasila, L., Velagapudi, V., Carroll, C.J., Auwerx, J., et al. (2014). Effective treatment of mitochondrial myopathy by nicotinamide riboside, a vitamin B3. EMBO Mol. Med. 6, 721-731.

Khan, N.A., Nikkanen, J., Yatsuga, S., Jackson, C., Wang, L., Pradhan, S., Kivelä, R., Pessia, A., Velagapudi, V., and Suomalainen, A. (2017). mTORC1 Regulates Mitochondrial Integrated Stress Response and Mitochondrial Myopathy Progression. Cell Metab. 26, 419428 e415.

Kotronen, A., Peltonen, M., Hakkarainen, A., Sevastianova, K., Bergholm, R., Johansson, L.M., Lundbom, N., Rissanen, A., Ridderstrale, M., Groop, L., et al. (2009). Prediction of nonalcoholic fatty liver disease and liver fat using metabolic and genetic factors. Gastroenterology 137, 865-872.

Kuhl, I., Miranda, M., Atanassov, I., Kuznetsova, I., Hinze, Y., Mourier, A., Filipovska, A., and Larsson, N.G. (2017). Transcriptomic and proteomic landscape of mitochondrial dysfunction reveals secondary coenzyme $Q$ deficiency in mammals. Elife 6, e30952.

Lehtonen, J.M., Försstrom, S., Bottani, E., Viscomi, C., Baris, O.R., Isoniemi, H., Höckerstedt, K., Österlund, P., Hurme, M., Jylhävä, J., et al. (2016). FGF21 is a biomarker for mitochondrial translation and mtDNA maintenance disorders. Neurology 87, 2290-2299.

Liu, L., Su, X., Quinn, W.J., 3rd, Hui, S., Krukenberg, K., Frederick, D.W., Redpath, P., Zhan, L., Chellappa, K., White, E., et al. (2018). Quantitative Analysis of NAD Synthesis-Breakdown Fluxes. Cell Metab. 27, 1067-1080 e1065.

Longo, R., Pollesello, P., Ricci, C., Masutti, F., Kvam, B.J., Bercich, L., Croce, L.S., Grigolato, P., Paoletti, S., de Bernard, B., et al. (1995). Proton MR spectroscopy in quantitative in vivo determination of fat content in human liver steatosis. J. Magn. Reson. Imaging 5, 281-285. Love, M.I., Huber, W., and Anders, S. (2014). Moderated estimation of fold change and dispersion for RNA-seq data with DESeq2. Genome Biol. 15, 550.

Migliavacca, E., Tay, S.K.H., Patel, H.P., Sonntag, T., Civiletto, G., McFarlane, C., Forrester, T., Barton, S.J., Leow, M.K., Antoun, E., et al. (2019). Mitochondrial oxidative capacity and NAD+ biosynthesis are reduced in human sarcopenia across ethnicities. Nat. Commun. 10, 5808.

Mudd, S.H., Brosnan, J.T., Brosnan, M.E., Jacobs, R.L., Stabler, S.P., Allen, R.H., Vance, D.E., and Wagner, C. (2007). Methyl balance and transmethylation fluxes in humans. Am. J. Clin. Nutr. 85, 19-25.

Nandania, J., Kokkonen, M., Euro, L., and Velagapudi, V. (2018a). Simultaneous measurement of folate cycle intermediates in different biological matrices using liquid 
chromatography-tandem mass spectrometry. J. Chromatogr. B. Analyt. Technol. Biomed. Life Sci. 1092, 168-178.

Nandania, J., Peddinti, G., Pessia, A., Kokkonen, M., and Velagapudi, V. (2018b). Validation and Automation of a High-Throughput Multitargeted Method for Semiquantification of Endogenous Metabolites from Different Biological Matrices Using Tandem Mass Spectrometry. Metabolites 8, E44.

Nikkanen, J., Försstrom, S., Euro, L., Paetau, I., Kohnz, R.A., Wang, L., Chilov, D., Viinamäki, J., Roivainen, A., Marjamäki, P., et al. (2016). Mitochondrial DNA Replication Defects Disturb Cellular dNTP Pools and Remodel One-Carbon Metabolism. Cell Metab. 23, 635-648.

Nunnari, J., and Suomalainen, A. (2012). Mitochondria: in sickness and in health. Cell 148, 1145-1159.

Pirinen, E., Canto, C., Jo, Y.S., Morato, L., Zhang, H., Menzies, K.J., Williams, E.G., Mouchiroud, L., Moullan, N., Hagberg, C., et al. (2014). Pharmacological Inhibition of Poly(ADP-Ribose) Polymerases Improves Fitness and Mitochondrial Function in Skeletal Muscle. Cell Metab. 9, 1034-1041.

Plaisance, E.P., Lukasova, M., Offermanns, S., Zhang, Y., Cao, G., and Judd, R.L. (2009). Niacin stimulates adiponectin secretion through the GPR109A receptor. Am. J. Phys. Endocrinol. Metab. 296, E549-558.

Rajman, L., Chwalek, K., and Sinclair, D.A. (2018). Therapeutic Potential of NAD-Boosting Molecules: The In Vivo Evidence. Cell Metab. 27, 529-547.

Ryu, D., Zhang, H., Ropelle, E.R., Sorrentino, V., Mazala, D.A., Mouchiroud, L., Marshall, P.L., Campbell, M.D., Ali, A.S., Knowels, G.M., et al. (2016). NAD+ repletion improves muscle function in muscular dystrophy and counters global PARylation. Sci. Transl. Med. 8, 361 ra139.

Shi, H., Enriquez, A., Rapadas, M., Martin, E., Wang, R., Moreau, J., Lim, C.K., Szot, J.O., Ip, E., Hughes, J.N., et al. (2017). NAD Deficiency, Congenital Malformations, and Niacin Supplementation. N. Engl. J. Med. 377, 544-552.

Spelbrink, J.N., Li, F.Y., Tiranti, V., Nikali, K., Yuan, Q.P., Tariq, M., Wanrooij, S., Garrido, N., Comi, G., Morandi, L., et al. (2001). Human mitochondrial DNA deletions associated with mutations in the gene encoding Twinkle, a phage T7 gene 4-like protein localized in mitochondria. Nat. Genet. 28, 223-231.

Spriet, L.L., and Whitfield, J. (2015). Taurine and skeletal muscle function. Curr. Opin. Clin. Nutr. Metab. Care 18, 96-101. 
Stefan, D., Di Cesare, F., Andrasescu, A., Popa, E., Lazariev, A., Vescovo, E., Strbak, O., Williams, S., Starcuk, Z., and Cabanas, M. (2009). Quantitation of magnetic resonance spectroscopy signals: the jMRUI software package. Meas. Sci. Technol. 20, 104035

Suomalainen, A., and Battersby, B.J. (2017). Mitochondrial diseases: the contribution of organelle stress responses to pathology. Nat. Rev. Mol. Cell Biol. 19, 77-92.

Suomalainen, A., Elo, J.M., Pietiläinen, K.H., Hakonen, A.H., Sevastianova, K., Korpela, M., Isohanni, P., Marjavaara, S.K., Tyni, T., Kiuru-Enari, S., et al. (2011). FGF-21 as a biomarker for muscle-manifesting mitochondrial respiratory chain deficiencies: a diagnostic study. Lancet Neurol. 10, 806-818.

Suomalainen, A., Majander, A., Haltia, M., Somer, H., Lönnqvist, J., Savontaus, M.L., and Peltonen, L. (1992). Multiple deletions of mitochondrial DNA in several tissues of a patient with severe retarded depression and familial progressive external ophthalmoplegia. J. Clin. Invest. 90,61-66.

Szczepaniak, L.S., Babcock, E.E., Schick, F., Dobbins, R.L., Garg, A., Burns, D.K., McGarry, J.D., and Stein, D.T. (1999). Measurement of intracellular triglyceride stores by $\mathrm{H}$ spectroscopy: validation in vivo. Am. J. Physiol. 276, E977-989.

Trammell, S.A., and Brenner, C. (2013). Targeted, LCMS-based Metabolomics for Quantitative Measurement of NAD+ Metabolites. Comput. Struct. Biotechnol. J. 4, e201301012.

Trammell, S.A., Weidemann, B.J., Chadda, A., Yorek, M.S., Holmes, A., Coppey, L.J., Obrosov, A., Kardon, R.H., Yorek, M.A., and Brenner, C. (2016). Nicotinamide Riboside Opposes Type 2 Diabetes and Neuropathy in Mice. Sci. Rep. 6, 26933.

Tyynismaa, H., Carroll, C.J., Raimundo, N., Ahola-Erkkilä, S., Wenz, T., Ruhanen, H., Guse, K., Hemminki, A., Peltola-Mjosund, K.E., Tulkki, V., et al. (2010). Mitochondrial myopathy induces a starvation-like response. Hum. Mol. Genet. 19, 3948-3958.

Van Bergen, N.J., Guo, Y., Rankin, J., Paczia, N., Becker-Kettern, J., Kremer, L.S., Pyle, A., Conrotte, J.F., Ellaway, C., Procopis, P., et al. (2019). NAD(P)HX dehydratase (NAXD) deficiency: a novel neurodegenerative disorder exacerbated by febrile illnesses. Brain 142, 50-58.

Van Goethem, G., Dermaut, B., Lofgren, A., Martin, J.J., and Van Broeckhoven, C. (2001). Mutation of POLG is associated with progressive external ophthalmoplegia characterized by mtDNA deletions. Nat. Genet. 28, 211-212. 
Vanhamme, L., van den Boogaart, A., and Van Huffel, S. (1997). Improved method for accurate and efficient quantification of MRS data with use of prior knowledge. J. Magn. Reson. 129, 35-43.

Vosper, H. (2009). Niacin: a re-emerging pharmaceutical for the treatment of dyslipidaemia. Br. J. Pharmacol. 158, 429-441.

Westphal, S., Borucki, K., Taneva, E., Makarova, R., and Luley, C. (2007). Extended-release niacin raises adiponectin and leptin. Atherosclerosis 193, 361-365.

Ylikallio, E., and Suomalainen, A. (2012). Mechanisms of mitochondrial diseases. Ann. Med. 44, 41-59.

Yoshino, J., Mills, K.F., Yoon, M.J., and Imai, S. (2011). Nicotinamide mononucleotide, a key $\mathrm{NAD}+$ intermediate, treats the pathophysiology of diet- and age-induced diabetes in mice. Cell Metab. 14, 528-536.

Zeviani, M., Servidei, S., Gellera, C., Bertini, E., DiMauro, S., and DiDonato, S. (1989). An autosomal dominant disorder with multiple deletions of mitochondrial DNA starting at the Dloop region. Nature 339, 309-311.

Zhang, H., Ryu, D., Wu, Y., Gariani, K., Wang, X., Luan, P., D'Amico, D., Ropelle, E.R., Lutolf, M.P., Aebersold, R., et al. (2016). NAD+ repletion improves mitochondrial and stem cell function and enhances life span in mice. Science 352, 1436-1443. 